\title{
La distinction par l'alimentation : Manger pour être (Moyen Âge et Siècle d'Or hispaniques)
}

\author{
Julia ROUMIER \\ Université Bordeaux Montaigne, Ameriber
}

RÉSUMÉ : Donnant une nouvelle impulsion à l'étude historique du comportement alimentaire et à l'anthropologie de l'alimentation, Jean Louis Flandrin fait de la "distinction par le goût » une clé d'interprétation majeure des pratiques du passé révélant la dimension politique des arts de la gastronomie. Cela permet en premier lieu de dépasser les visions simplistes d'un Moyen Âge rustre, aux manières grossières, essentiellement consommateur de viandes rôties et utilisant les épices sans finesse. Cette gastronomie est en réalité une rhétorique élaborée et ritualisée et ces aspects alimentaires de l'ostentation sont bien des signes de supériorité, au sens de ce que J.P. Daloz (2010) nomme des symboles matériels ou « prestigious goods», une spectacularisation de la domination à travers une expression sensorielle mais aussi une source de plaisir physique codifiée dans un environnement courtisan : la table est bien un espace politique.

Non sans raison le roi Alphonse X, dans le Titre VII des Siete partidas, dédié à l'éducation, offre une place importante à des lois régissant le comportement à table, lieu de plaisir, de réunion et de discussion. La nourriture est au cœur des relations humaines et, en particulier à la cour et dans les sphères du pouvoir se nouent autour de la table les jeux de représentations structurant la hiérarchie sociale et consacrant la légitimité des puissants.

Mots-clés : Alimentation, Distinction, Etiquette, Moyen Âge, Espagne

RESUMEN: Dando un nuevo impulso al estudio histórico de los comportamientos alimentarios y a la antropología de la alimentación, Jean Louis Flandrin hace de la "distinción por el gusto" una clave importante para interpretar las prácticas del pasado, revelando la dimensión política de las artes gastronómicas. Esto nos permite ir más allá de las visiones simplistas de una Edad Media grosera, con modales toscos, consumiendo esencialmente carnes asadas y utilizando especias sin delicadeza. Esta gastronomía es, de hecho, una retórica elaborada y ritualizada, y estos aspectos alimentarios de ostentación son, en efecto, signos de superioridad, en el sentido de lo que J.P. Daloz (2010) llama símbolos materiales o " prestigious goods ", una espectacularización de la dominación a través de una expresión sensorial, pero también una fuente de placer físico codificado en un entorno cortesano: la mesa es, en efecto, un espacio político.

No sin razón, el rey Alfonso X, en el Título VII de las Siete partidas, dedicado a la educación, da protagonismo a las leyes que regulan el comportamiento en la mesa, lugar de placer, reunión y discusión. La comida está en el centro de las relaciones humanas y, sobre todo en la corte y en las esferas del poder, los juegos de representación que estructuran la jerarquía social y consagran la legitimidad de los poderosos se desarrollan en torno a la mesa.

Palabras claves: Alimentación, Distinción, Etiqueta, Edad Media, España

ABSTRACT: Giving new impetus to the historical study of eating behaviour and to the anthropology of food, Jean Louis Flandrin makes "distinction by taste" a major key to interpreting past practices, revealing the political dimension of the arts of gastronomy. This allows us to go beyond simplistic visions of a boorish Middle Ages, with coarse manners, essentially consuming roasted meats and using spices without finesse. This gastronomy is in fact an elaborate and ritualised rhetoric and these food aspects of ostentation are indeed signs of superiority, in the sense of what J.P. Daloz (2010) calls material symbols or 'prestigious goods', a spectacularisation of domination through a sensory expression but also a source of codified physical pleasure in a courtier environment: the table is indeed a political space.

Not without reason, King Alfonso X, in Title VII of the Siete partidas, dedicated to education, gives prominence to laws governing behaviour at the table, a place of pleasure, meeting and discussion. Food is at the heart of human relations and, in particular at court and in the spheres of power, games of representation are played around the table, structuring the social hierarchy and establishing the legitimacy of the powerful.

Keywords: Food, Distinction, Etiquette, Middle Ages, Spain 
Donnant une nouvelle impulsion à l'étude historique du comportement alimentaire et à l'anthropologie de l'alimentation, Jean Louis Flandrin (Flandrin, 1986, p. 267-309) fait de la « distinction par le goût » une clé d'interprétation majeure des pratiques du passé révélant la dimension politique des arts de la gastronomie. Cela permet en premier lieu de dépasser les visions simplistes d'un Moyen Âge rustre, aux manières grossières, essentiellement consommateur de viandes rôties et utilisant les épices sans finesse (Montanari, 2015). Les travaux fondateurs de Bruno Laurioux ont combattu ces préjugés (Laurioux, 2002 ; Laurioux et Horard-Herbin, 2017 ; Laurioux, Paravicini-Bagliani, Pibiri, 2018) et d'autres ont souligné le rôle de l'étiquette, des arts de la table et des épices dans des pratiques médicales et culinaires raffinées (Freedman, 2010 ; Garrido Aranda, Hidalgo Nuchera et Ramírez Ponferrada, 2004), un sujet qui est aujourd'hui l'objet d'un intérêt renouvelé comme le montrent de récentes publications (Quellier, 2021). Cette gastronomie est en réalité une rhétorique élaborée et ritualisée et ces aspects alimentaires de l'ostentation sont bien des signes de supériorité, au sens de ce que J.P. Daloz (2010) nomme des symboles matériels ou "prestigious goods », une spectacularisation de la domination à travers une expression sensorielle mais aussi une source de plaisir physique codifiée dans un environnement courtisan : la table est bien un espace politique (Campanini, 2019).

Non sans raison le roi Alphonse X, dans le Titre VII des Siete partidas, dédié à l'éducation, offre une place importante à des lois régissant le comportement à table, lieu de plaisir, de réunion et de discussion. La nourriture est au cœur des relations humaines et, en particulier à la cour et dans les sphères du pouvoir se nouent autour de la table les jeux de représentations structurant la hiérarchie sociale et consacrant la légitimité des puissants. Le roi sage se base sur un ouvrage de prestige de cent cinquante ans son prédécesseur, la Disciplina clericalis de Pedro Alfonso. La loi V en particulier rappelle l'importance de l'esthétique et de l'élégance au moment de manger et de la nécessité pour les précepteurs d'éduquer leur pupille sur ce point, " apuestadamente ", la distinction à table est un apprentissage où l'ignorance est une grossièreté indigne des puissants. L'élégance et l'esthétique des pratiques alimentaires est donc bien un puissant marqueur social dont les textes normatifs médiévaux s'emparent consciemment pour le réguler et en fixer les normes. La fin du Moyen Âge marque en effet en Occident l'initiation d'un "processus de civilisation » (Thomas, 2003), avec l'établissement progressif de règles de civilités de plus en plus contraignantes et envahissantes (Alvar et Alvar-Nuño, 2020). C'est par exemple le développement des manuels de bienséances et des manières de table, ainsi que toutes les facettes du " souci de soi » issu de l'idéal courtois et débouchant sur une urbanitas, évidemment liée au monde urbain. On observe ainsi au XVe siècle en Castille les débuts de l'imposition de l'étiquette, avec une civilité raffinée, le développement de la rédaction de 
recueils de recettes, mais également les variations de modes permettant de marquer la supériorité du roi par le rythme et les codes qu'il impose à sa cour (Allard, 1995 ; voir aussi Riera i Melis, 2015). Les différentes facettes des arts de la table et de la gastronomie sont ainsi le champ où se construit l'identité nobiliaire, l'instrument d'une ritualisation de sa supériorité (Miguel Prendez, 2003) qui intègre des éléments tirés de la littérature pour mieux fictionnaliser la mise en scène de la distinction (Assis González et Chiappero, 2017). Pour comprendre les structures de domination en jeu, nous aurons à nous référer au concept de distinction élaboré par Pierre Bourdieu. Ce concept sociologique délimite les signes et pratiques permettant une différenciation élitaire dans une société stratifiée mais où une certaine mobilité est possible (Bourdieu, 1979), ce qui correspond au cadre social de l'étude. La consommation ostentatoire est donc l'expression d'une esthétique définie par les élites et différencie ainsi ceux qui savent dépenser l'argent avec goût de ceux qui manquent de la formation culturelle appropriée. Par goût nous entendons bien le « bon goût » tel que Pierre Bourdieu en a fait l'analyse, le révélant comme critère de distinction élitaire, outil d'une injonction normative. Dans La Distinction, Pierre Bourdieu démonte en effet l'idée commune du goût comme faculté de discernement, pour démontrer qu'il s'agit bien davantage d'une pratique codifiée par les élites et suscitant des réactions attendues par la société (Koch 2013).

L'alimentation serait un des outils d'affirmation de la distinction (Lenoir, 2014), capital symbolique proche de ce que Weber appelait le charisme, concept réunissant le prestige, la réputation et l'autorité. Une de ses spécificités est de se manifester dans la dénégation de l'économie au sens ordinaire, comme pour l'« effet Veblen ». Cela correspond en particulier à l'économie du don (Marcel Mauss), qu'on peut appliquer à la mise en scène codifiée la générosité par la royauté ou la noblesse : le puissant redistribue pour montrer sa largesse et ses faveurs. L'alimentation ne suit pas non plus de simples critères cartésiens en consommant toute ressource accessible car dans ce domaine s'exerce bien un puissant arbitraire culturel du comportement commensal (Garine, 2007, p. 53). Le repas est un spectacle où le service joue un rôle essentiel : les principaux rôles de service sont attribués comme des honneurs à des membres importants de la noblesse et le plan de table reflète les rapports de force. La vigueur des « querelles de préséance épulaire » rappelle l'importance symbolique du moment de la consommation alimentaire (Laurioux, 1992). Chacun se déplace dans une chorégraphie précise et signifiante qui fait partie du divertissement proposé, leurs mouvements exacts et gracieux, dans un ballet qui marque la préséance du maître en multipliant les déplacements autour de sa position centrale et dominante, sur une estrade, sous un dais... vers lui les regards convergent tout comme les gestuelles des 
personnels et des convives. Ce personnel est en outre régi par une hiérarchie codifiée : les fonctions de maestresala mayor ou de repostero de estrados sont ainsi un honneur réservé aux plus grands personnages.

Martina Magali Díaz Sammaroni (2019) a ainsi souligné l'importance nouvelle acquise par cette dernière charge avec la dynastie Trastámara, à partir 1369, moment où se produit une grande rénovation des charges administratives et palatiales. Parmi les officiers au service du roi et de la cour s'affirmait en particulier le « repostero de estrados » dont le travail consistait à préparer les pièces et décors pour la présence du roi, à la différence du repostero de plata, chargé de la disposition des plats à la table princière. Le respostero devait également être vêtu de la façon la plus luxueuse, comme le recommande par exemple Fernández de Oviedo, ajoutant qu'il vaut mieux qu'il ne soit ni petit, ni laid, ni contrefait : «

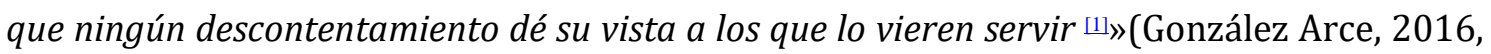
469); preuve de l'importance de l'esthétique dans les signes du pouvoir jusque dans le service de la table.

Les rituels entourant le service, les préséances dans le placement, le décor et la vaisselle, les techniques (comme la découpe), les gestes avec la présentation des plats et boissons construisent un discours exprimant le statut de chacun, faisant de la cérémonie alimentaire une véritable science (Gascón Vera, 2016). Plus le rang est élevé, mieux on mange. L'entremet est ainsi un plat honorifique, réservé à la première table, la plus digne ; le rôtissage est le mode de cuisson le plus prestigieux sur lequel s'exerce en outre l'activité prestigieuse de l'écuyer tranchant ; les tripes sont réservées au dauphin, les volailles aux puissants (Laurioux, 1992). Mais comme le souligne Antoni Riera i Melis (2015), la consommation alimentaire se distingue des autres produits ostentatoires : l'apparat du décor contribue à une image projetée vers l'extérieur, ouverte aux regards. Mais, de façon spécifique, la consommation alimentaire, les saveurs et arômes, sont une consommation internalisée, une ingestion, et étaient destinés à un cercle plus restreint et atteignaient les personnes par un faste plus intime. Le visuel est toutefois essentiel pour donner le faste voulu au repas et le service doit donc organiser la distribution comme une célébration ostentatoire. La présentation des aliments est un spectacle avec les « aparadores », meubles servant à présenter les plats, les pièces de mobilier de service et la vaisselle. Or, argent, l'emploi de matériaux précieux rappelle ceux des calices et custodes et la mise en scène des aparadores ne manque pas de ressembler à celle d'un maître autel. Reflet de l'eucharistie, la mise en scène de l'alimentation s'inscrit dans de complexes réseaux symboliques et esthétiques. Comme l'écrit ainsi María de los Ángeles Pérez Samper : « El lujo no residía sólo en los alimentos propiamente dichos, sino en el entorno. Complementario a comer bien era 
decorar el plato, la mesa y el comedor » (Pérez Samper, 1998, p. 58) ${ }^{[2]}$ Et nous devrons donc prêter attention au décor de la table, à la vaisselle et au service, sans nous étonner que les sources y aient été plus sensibles qu'aux ingrédients, recettes ou saveurs des plats eux-mêmes.

Certains travaux ont en effet pu souligner l'envahissement du critère visuel dans les pratiques alimentaires de prestige ; l'aspect et la dimension spectaculaire paraissent dans bien des sources primer sur la saveur du plat, faut-il en déduire la primauté du visuel sur le goût, en considérant la similarité des usages vestimentaires et alimentaires (Vincent-Cassy, 1996). Cette importance du visuel dans l'art culinaire s'accorde surtout à l'art visuel de l'écriture, avec des descriptions reflétant ces mises en scène : mais quelle place dans les textes pour l'appréciation du goût et des saveurs ? et qu'en déduire de la réalité des pratiques?

Vanter les plaisirs de l'art de la table n'a rien d'anodin car la gourmandise et la gloutonnerie sont des vices condamnés par les discours religieux. Comment alors louer les mets servis à une table sans pour autant faire passer son hôte pour un glouton gouverné par ses passions ? Citons Diego Enríquez del Castillo (Sánchez Martín, 1994, p. 135) décrivant Henri IV de Castille : «Su comer más fue desorden que glotiana, por donde su complisyón antiguamente se corrompió. Nunca jamas bevió vyno ». Il faudrait analyser la conciliation de cette visée somptuaire de l'alimentation ostentatoire avec les normes religieuses et les cadres du Carême. Cela pose problème en particulier pour le vin, boisson centrale de la culture chrétienne et des rituels nobiliaires (Framiñán Santas, 2005), mais aussi pour la viande, fortement consommée dans la société aristocratique, soumise aux restrictions des périodes de jeunes et carêmes, et dont la signification symbolique était à la fois une affirmation de statut (privilège de chasseur) et une appropriation païenne de la force de l'animal (Montanari, 1983, p. 57) ; «the ideal strength-giving food » (Montanari, 2015 : 75) étant spiritualisée par la consommation privilégiée des oiseaux.

L'espace de la péninsule Ibérique est le lieu de rencontres culturelles et interreligieuses particulièrement intéressant pour le sujet de l'alimentation ${ }^{[3]}$. Nombres d'ingrédients orientaux ou de pratiques gastronomiques exotiques entre en chrétienté par cette porte. Alors, que mange-t-on à ces belles tables aristocratiques où tout est fait pour exalter la grandeur de l'hôte ? Au Moyen Âge, l'abondance et la diversité des plats sont les deux caractéristiques permettant d'exprimer la magnificence d'un banquet (Castro Hernandez, 2019, p. 180), la profusion est symbole de générosité et de grandeur. En particulier on trouve comme critère d'éloge la diversité des techniques de préparation ou la superposition de saveurs avec les plats sucrés-salés ou aigres-doux. Le sucre, élément ostentatoire signifiant le raffinement et donc l'estime portée à l'hôte, détrône le miel pour adoucir les 
préparations culinaires des élites à la fin du bas Moyen Âge ${ }^{[4]}$. Le sucre a permis le développement de diverses confitures et confiseries, cadeaux précieux ; il était en outre considéré comme une épice pour ses qualités thérapeutiques, toniques ou propitiatoires (Diaz Sammaroni, 2020, p. 188). Au-delà de la dimension médicale, intimement mêlée aux pratiques alimentaires (Laurioux, 2006), il s'agit bien de pratiques culturelles basées sur le souci de légitimation statutaire par la consommation de mets onéreux voire pourvu d'une symbolique sacrée comme le sucre et les épices dont la saveur était celle du sacré et du Paradis comme l'affirment très tôt les Etimologías d'Isidore de Séville (Laurioux, 1993).

Ces nombreuses questions s'entrelacent autour de l'alimentation au service de la Distinction pour les périodes du Moyen âge et du Siècle d'Or dans les espaces ibériques. Elles nous permettent également d'aborder la spectacularisation du pouvoir, l'art de la médecine, la circulation du savoir et des modes, le paysage sensoriel et le plaisir face aux discours normatifs de l'Eglise... l'étude conjointe de l'alimentation et de la distinction offre ainsi un riche champ exploratoire, depuis la matérialité concrète des aliments et de leurs préparations, jusqu'aux modalités de répartition ou dégustation, les symboliques et les rituels des arts de la table et de la sociabilité alimentaire.

\section{Apports du dossier}

Le premier axe du dossier est consacré à la distinction en rapport à la dimension diététique et à l'hygiène. L'alimentation est en effet indissociable de la médecine, dans la régulation des humeurs et l'équilibre apporté au mangeur en fonction de son profil. Serrano Larrayoz, Fernando (Université de Alcala) offre une réflexion sur les conseils diététiques (nourriture et boisson) appropriés pour maintenir la santé d'un personnage appartenant à l'élite ecclésiastique de la fin du XIVe siècle. Il analyse pour cela le troisième « consiliatorio » de la première partie du traité intitulé Libro de visitaçione e conssiliaçione medicarum (ca. 1381), une œuvre érudite, nourrie à plus d'une trentaine de sources savantes. Écrite dans un castillan traversé de termes latins, elle est marqué par un fort anti-judaisme que révèle sa défense de la consommation de viande de porc pour un destinataire qui pourtant aurait bénéficié de réduire la part carnée de son alimentation. Cette étude permet donc plus largement d'étudier cette opposition entre l'alimentation carnée, riche et épicée propre à célébrer la grandeur d'un personnage de l'élite, et les besoins de régulation diététique qu'imposent ses excès et les maladies induites.

Hélène Jawhara Piñer (U. de Tours, CESR (UMR7323 of the CNRS), s'intéresse quant à elle à la diététique en relation avec les plaisirs de la table dans le premier livre de cuisine connu de la péninsule ibérique le Kitab al tabīhl[Le livre de cuisine]. Cela lui permet de réfléchir à 
l'articulation de deux préoccupations qui paraissent s'opposer : Le plaisir de manger étaitil compatible avec une alimentation saine ? Les livres de cuisine andalous, principalement dirigés à une élite sociale, privilégient des ingrédients onéreux permettant de souligner leur richesse. Mais les épices employées avec abondance sont aussi les ingrédients des remèdes et soins préconisés par les traités médicaux. Au final, le plaisir de bien manger se révèle comme la meilleure protection contre les maladies.

La réflexion de Sophie Coussemacker (U. Bordeaux Montaigne) nous amène dans l'espace bien insuffisamment traité de la cuisine elle-même, là où s'active une brigade nombreuse et diversifiée : la distinction est en effet également de mise là où l'alimentation s'élabore et s'applique, dans une certaine mesure, à ceux qui réalisent les préparations. Il s'agit là non seulement de garantir la propreté par égards pour les consommateurs, mais aussi de construire la dignité des professionnels de l'alimentation, alors même que le cuisinier n'est guère valorisé dans un certain nombre de sources : «L'élégance du cuisinier. Entre préceptes hygiénistes et distinction : la propreté en cuisine dans le Libre del coch de Robert de Nola $(1477,1520,1529)$ ». Ce métier manuel, en prise avec la matière même de l'alimentation et avec le feu de la cuisson peut en effet être salissant et cela a contribué à en donner une image dégradée. La distinction de l'hôte s'incarne donc dans la recherche d'une extrême propreté dans les cuisines et dans la dignité du cuisinier qui seules peuvent permettre de satisfaire pleinement aux exigences d'un consommateur raffiné. L'article propose de nous centrer sur le le Libre del coch de Robert de Nola $(1477,1520,1529)$ mais fournit aussi des points de comparaison tirés d'autres sources afin de saisir la force de cette injonction à la perfection du cuisinier, entre normes et réalité d'un office au plus près des puissants.

Le second axe de ce dossier nous offre une réflexion approfondie sur les modalités d'expression du pouvoir à table :

Moins qu'un lieu de plaisir, la table est un lieu d'éducation au pouvoir et un moyen de l'exercer, surtout évidemment dans les ouvrages normatifs comme on peut le voir dans certains manuels et « miroirs » princiers ou royaux (Martínez Alcorlo, 2016). L'étude menée par Nathalie Peyrebonne (LECEMO, U. Sorbonne Nouvelle), sur le traité Nutrición real de González de Salcedo, publié en 1671, révèle qu'au XVIe siècle, plus que jamais, les règles de sociabilité s'affinent et sont diffusées, dans des manuels de bonnes manières révélateurs de la force idéologique de cette distinction par l'alimentation. Il s'agit ici d'un manuel d'éducation destinés aux enfants royaux âgés de 7 à 14 ans et dur vingt-sept chapitres, sept sont intégralement consacrés à la façon dont il leur faudra se nourrir. Les consignes révèlent l'importance de la propreté et de la maitrise du temps : il ne faut pas de précipitation dans la consommation alimentaire, mais surtout le souverain doit viser la recherche de la beauté 
et de l'élégance, montrer son aisance et bannir toute vulgarité. C'est un lieu de conversation où la dignité doit être maintenue scrupuleusement, il siège à table comme sur son trône.

Davantage intéressé par la dimension matérielle de ce faste commensal, David Nogales Rincón (U. Autónoma de Madrid) nous offre une réflexion sur l'ensemble des comportements et rituels du banquet à la fin du moyen âge. Son analyse très complète des objets et rituels du faste alimentaire met en valeur une rhétorique qui reflète les influences entre les cours. L'intégration des différents sens, au-delà de la vue, l'odorat, le goût, l'ouïe, est recherchée par une théâtralisation de l'art de la table qui articule aussi la musique et le littéraire.

En parfait complément à cette réflexion, Diana Pelaz Flores (Université de Saint-Jacques de Compostelle) s'intéresse aux formes féminines de cette ostentation alimentaire. La question comportementale invite ainsi à réfléchir à la notion de frugalité. La spectacularisation de l'alimentation de la reine détermine un soin particulier à sa célébration comme rituel, selon des stratégies distinctions des modalités masculines que révèlent les sources écrites.

Marc Zuili (U. de Versailles Saint-Quentin-en-Yvelines) s'intéresse quant à lui au lexique abondant de l'alimentation dans le Trésor de César Oudin paru en 1607 afin d'y révéler un tableau exact et très complet des us et coutumes alimentaires pratiqués alors dans ce pays, ainsi que leur perception en France. On y découvre ainsi les lieux, usages et objets de l'alimentation, les métiers de bouche, aussi bien que les modes de cuisson et ustensiles employés.

Mehdi Gourghiate (U. Bordeaux Montaigne) s'intéresse ici à la façon dont les califes almohades devaient apparaître comme les promoteurs du merveilleux, à travers leur cuisine. Il démontre ainsi l'importance de la prohibition des anciens usages communautaires de vin cuit (anzīr) au profit d'une consommation réservée à l'élite au pouvoir. Ce faisant, les sources rendirent compte de cette inflexion en utilisant un terme arabe aussi flou qu'ambigu le rubb lequel ne faisant pas nécessairement référence à un boisson alcoolisée. Entre prohibition du vin et nécessité d'affirmer son rang en se distinguant de ses sujets, les califes almohades firent savoir qu'ils avaient le privilège de consommer du rubb soit un terme suffisamment ambigu. Plus encore, ils étaient en mesure de faire couler des rivières de $r u b b$ et de rappeler qu'ils pouvaient faire entrevoir à leurs sujets un éden terrestre qu'eux seuls étaient à même de réaliser.

Cet éden de plaisirs qu'est la nourriture au service de la démonstration de pouvoir est le fil directeur de ce volume : nous espérons qu'il est porteur de riches réflexions les différentes facettes de ces arts de la table au plus près du trône, incitant à de nouvelles lectures de cette 
stratégie de conviction et de légitimation qui passe par les plaisirs de la chair et leur théâtralisation. 


\section{Bibliographie}

ALLARD Jeanne, «La naissance de l'étiquette : les règles de vie à la Cour de Castille à la fin du Moyen Âge », in Nilda Guglielmi et Adeline Rucquoi (dirs.), El discurso político en la Edad Media, Buenos Aires : Consejo nacional de investigaciones científicas y técnicas ; Paris : CNRS, 1995, 11-28.

AlVAR Carlos et Alvar-NuÑo Guillermo, Normas de comportamiento en la mesa durante la Edad Media, Sial/Trivium, Madrid, 2020.

ASISS GONZÁLEZ Federico J. et Luciana CHIAPPERO, «El banquete y la identidad noble. Su representación en la literatura de la Baja Edad Media (S.XIV) », in RIVAR, vol. 4, n¹1, 2017, 74-91.

BouRdiEu Pierre, La distinction, critique sociale du jugement, Paris, Minuit, 1979.

CAMPANELli Antonella, Food Cultures in Medieval Europe. Traduit par Leah M. Ashe, Bruxelles, Berlin, Bern, New York, Oxford, Peter Lang Edition, 2019.

CASTRO HERnANDEZ Pablo, "Comidas especias y practicas alimentarias en los viajes medievales a Oriente. Siglos 13-15 », in Viajeros en China y libros de viajes a Oriente, Rafael Beltran (dir.), U. de Valencia, 2019, 175-201.

DALOZ Jean-Pascal, The sociology of Elite Distinction. From Theoretical to Comparative Perspectives, Palgrave, Macmillan, 2010, 77-80, chap. 4 : External Signs of Superiority.

DiaZ SAMmARoNi Martina Magali « Las fiestas castellanas bajomedievales a traves de los sentidos : el caso de Don Miguel d'Iranzo », in Hispania-Espanna-Españas. Diálogos y dinámicas históricas en el mundo hispánico, éd. Ezequiel Borgognoni et Manuel Rivero Rodríguez, 2020, 153-193, 188.

Diaz SAmmaroni Martina Magali, «El modelo sensorial festivo de Juan II de Castilla (1406-1454), in Senses and sensibilities in classical and medieval worlds. Gerardo Fabián Rodríguez, Gisela $\begin{array}{lllll}\text { Coronado Schwindt } \quad \text { (coord.) } & \text { Mirabilia, 29, 2019/2, }\end{array}$ https://www.revistamirabilia.com/issues/mirabilia-29-2019-2

FLANDRIN Jean-Louis, « La distinction par le goût » in Aries, Duby (dir.), Histoire de la vie privée, t. 3, De la Renaissance aux lumières, Paris, Seuil, 1986, 267-309.

Cruz Cruz Juan, Dietética medieval. Apéndice con la versión castellana del "Régimen de salud" de Arnaldo de Vilanova. Huesca, La Val de Onsera, 1997.

FRAMIÑÁN SANTAS Ana María, «El vino y la nobleza en la Edad media : alimentación y cortesía », in Actas de la cultura del vino : primer congreso peninsular, coord. Isidro García Tato, Ana María Suárez Piñeiro, 2005, 67-94.

FREEDMAN Paul, Lo que vino de oriente : las especias y la imaginación medieval, Salustiano Moreta y José Ramón Gutiérrez (trad.), Universitat de Valencia, Valencia, 2010.

Garrido Aranda Antonio, Hidalgo Nuchera Patricio e Ramírez Ponferrada María Dolores, « Papel de hierbas aromáticas y especias en la alimentación española de los tiempos modernos », in A. Garrido aranda (comp.): El sabor del sabor: hierbas aromáticas, condimentos y especias, Córdoba, Universidad de Córdoba, 2004, 140-149.

GARINE Igor de, 2007 - «Remerciement de M. Igor de Garne. A propos d'ethnologie », in Académie de Béarn, Annales, 2005. Biarritz, Atlantica, 45-59.

GASCÓN VERA Elena, « La ceremonia como ciencia: "El Arte cisoria" de Enrique de Villena », Alicante : Biblioteca Virtual Miguel de Cervantes, 2016: Edición digital a partir de Actas del octavo Congreso de la Asociación Internacional de Hispanistas : celebrado en Brown University, Providence Rhode Island, del 22 al 27 de agosto de 1983, Madrid, Ediciones Istmo, 1986, 587595.

GoNZALEZ ARCE José Damián, La casa y corte del príncipe don Juan (1478-1497) : economía y etiqueta en el palacio del hijo de los Reyes Católicos, Séville, sociedad española de estudios medievales, 2016, 469.

Gounelle Rémi, Anne-Laure Zwilling et Yves Lehmann, éd. Religions et alimentation. Normes alimentaires, organisation sociale et représentations du monde, Turnhout, Brepols, 2019. 
Horard-Herbin Marie-Pierre et Bruno Laurioux, Pour une histoire de la viande. Fabrique et représentations de l'Antiquité à nos jours, Presses Universitaires de Rennes et François Rabelais, 2017.

KосH Erec R, «La pratique du goût : de Pierre Bourdieu à Antoine de Courtin », in Dix-septième siècle, 2013/1 (n² 258), 45-54. DOI: 10.3917/dss.131.0045. URL: https://www.cairn.info/revue-dixseptieme-siecle-2013-1-page-45.htm

LAURIOUX Bruno, Le Banquet. Manger, boire et parler ensemble (XII - XVII siècles), éd. Bruno Laurioux, Agostino Paravicini-Bagliani et Eva Pibiri, Florence, Edizioni del Galuzzo, 2018.

"Cuisine et médecine au Moyen Âge », in Cahiers de recherches médiévales [En ligne], 13 spécial | 2006, mis en ligne le 03 avril 2009, consulté le 13 octobre 2020. URL : http:// journals.openedition.org/crm/862 ; DOI : https://doi.org/10.4000/crm.862

comme Manger au Moyen Âge. Pratiques et discours alimentaires en Europe aux xive et $x v^{e}$ siècles, Paris, Hachette, 2004.

Vins musqués et flaveurs de paradis : l'imaginaire médiéval des épicesin : Le Monde végétal (XIIe-XVIIe siècles): Savoirs et usages sociaux [en ligne]. Saint-Denis: Presses universitaires de Vincennes, 1993 (généré le 10 août 2021). Disponible sur Internet: <http://books.openedition.org/puv/7963>. ISBN : $9782379241253 . \quad$ DOI : https://doi.org/10.4000/books.puv.7963.

«Table et hiérarchie sociale à la fin du Moyen Âge », in Lambert Carole (dir.), Du manuscrit à la table. Essais sur la cuisine au Moyen Âge et répertoire des manuscrits médiévaux contenant des recettes culinaires, Montréal-Paris, Les Presses de l'Université de Montréal \& Champion-Slatkine, 1992, 98-100.

LENOIR Rémi, « Noblesse et distinction sociale dans l'œuvre de Pierre Bourdieu », p. 21, in Marquer la prééminence sociale, le pouvoir symbolique en Occident. 1300-1640. Jean-Philippe Genet et F. Igor Mineo, Sorbonne et école française de Rome, 2014.

MARTinez AlCORLo Ruth, «Un curioso speculum reginae para la joven Isabel : Criança y virtuosa dotrina de Pedro Gracia Dei (ca. 1486) », Memorabilia, Número 18 (2016), 204-234.

Miguel-Prendes Sol, « Chivalric identitity in Enrique de Villena's Arte Cisoria », in La Corónica, 32.1, 2003, 307-42.

Millán Fuertes A.A. (éd.) Arbitrario cultural. Raciolanidad e irracionalidad del comportamiento comensal. Homenaje a Igor de Garine. Huesca, La Val de Onsera, 2004.

Montanari Massimo, Medieval Tastes. Food, Cooking and the Table, Traduction par Beth Archer Brombert, Columbia University Press, New York, 2015.

Valeurs symboles messages alimentaires durant le haut moyen âge » Médiévales, $\mathrm{n}^{\circ}$ 5, 1983, 57-66.

OUERFELLI Mohamed, « Le banquet en France et en Italie à la fin du Moyen Âge : entre convivialité et propagande », in Dynamiques sociales au Moyen Âge en Occident et en Orient, É. Malamut éd., Aix-en-Provence, Presses universitaires de Provence, 2010, 117-134.

PÉREZ SAMPER María de los Ángeles, La alimentación en la España del Siglo de Oro. Domingo Hernández de Maceras, Libro del Arte de Cocina, Huesca, La Val de Onsera, 1998.

QUELLIER Florent, Histoire de l'alimentation ; de la Préhistoire à nos jours. Belin, 2021.

RIERA I MELIS Antoni, « Jerarquía social y desigualdad alimentatia en el Mediterráneo Noroccidental en la Baja Edad Media. La cocina y la mesa de los estamentos privilegiados », in Acta histórica et archeológica mediaevalia, n¹6-17, 19095-1996, 181-205.

«Dulzores orientales para traumas postpestilenciosos : importaciones de azúcar en Barcelona durante el bienio de 1349-1350", in Mercados de lujo, mercados del arte : el gusto de las élites mediterráneas en los siglos XIV y XV, Brouquet, Sophie et Juan Vicente García Marsilla (dir.), 2015, 465-491.

RIERA I MELIS Antoni, « Gastronomía y política en los banquetes cortesanos de la baja edad media », in M. Garcia Guatas, La alimentación en la corona de Aragon, 2013, 65-100. 
SÁnchez Martin Aureliano (éd. crit. ), Diego Enriquez del Castillo, Crónica de Enrique IV de Castilla, Universidad de Valladolid, 1994.

THomas Jérôme, Corps violents, corps soumis: le policement des moeurs à la fin duMoyenÂge, Paris, L'Harmattan, 2003.

Thomasik Timothy J. et Juliann M. Vitullo (éd.), At the table. Metaphorical and material culture of food in Medieval and Early Modern Europe, Turnhout, Brepols, 2007, p. 145-166.

VINCENT-CASSY Mireille, « La vue et les mangeurs : couleurs et simulacres dans la cuisine médiévale », I, Banquets et manières de table au Moyen Âge, Centre universitaire d'études et de recherches médiévales d'Aix, Sénéfiance n³8, 1996, 159-176. 


\section{Notes}

[1] Gonzalez Arce, José Damián, La casa y corte del príncipe don Juan (1478-1497) : economía y etiqueta en el palacio del hijo de los Reyes Católicos, Séville, sociedad española de estudios medievales, 2016, p. 469.

[2] Voir aussi : Riera Melis (2013, p. 65-100) : « La concentración de la riqueza permitió a las elites de los siglos XIV y XV manifestar su elegancia acentuando el refinamiento en la mesa, donde regían las "buenas costumbres" y la presentación de los platos se valoraba tanto como el contenido ».

[3] Pour la gastronomie espagnole à la période moderne, envisagée en incluant le XV siècle, je recommande la magnifique bibliographie répertoriée en 2018 par Lourdes Gutiérrez et Antonio Rodríguez Vela pour la Biblioteca Nacional de España.

http://www.bne.es/es/Micrositios/Guias/gastronomia/resources/pdf/GASTRONOMIA ESPAxOL A.pdf

[4] Deux thèses importantes ont été consacrées au sucre médiéval : Mohamed Ourfelli, Le sucre, production, commercialisation et usages dans la Méditerranée Médiévale, Leiden-Boston, 2008 et Adela Fábregas, Producción y comercio del azúcar en el Mediterráneo medieval. El ejemplo del reino de Granada, Granada, 2000. 